\title{
Coronary air embolism treated with aspiration catheter
}

\section{S Patterson, F Kiemeneij}

Heart 2005;91:e36 (http://www.heartinl.com/cgi/content/full/91/5/e36). doi: 10.1136/hrt.2005.060129

\begin{abstract}
Coronary air embolism remains a recognised complication of coronary catheterisation despite a strong emphasis on prevention. Current treatment consists of supportive measures with 100\% oxygen and analgesia. Recent case reports describe the use of mechanical treatments aimed at dispersing or removing the air embolus with variable success. A case of coronary air embolism causing an acute coronary syndrome is described that was definitively treated with an aspiration system. The effectiveness of the aspiration system in the distal section of an obtuse marginal artery indicates that such dedicated aspiration systems may prove useful in the standard treatment of air embolism.
\end{abstract}

C oronary air embolism is a recognised complication of cardiac catheterisation in $0.1-0.3 \%$ of cases. ${ }^{12}$ Sequelae range from a clinically insignificant event to an acute coronary syndrome ${ }^{3}$ and death. ${ }^{4}$ Treatment has consisted mainly of supportive measures, the use of $100 \%$ oxygen (to minimise ischaemia and to establish a diffusion gradient encouraging elimination of gas from the bubble), and pain relief. Mechanical interventions have been reported such as attempts at dispersal ${ }^{25}$ or use of non-dedicated equipment for aspiration. ${ }^{36}$ We describe a case where intervention through the radial artery with a dedicated aspiration catheter completely resolved the clinical and angiographic changes of an acute coronary syndrome due to an air embolus lodged distally in a large obtuse marginal vessel.

The Medtronic Export aspiration catheter (Medtronic) is a 4 French monorail system with a $1 \mathrm{~mm}$ (0.04l inch) lumen that is continuous with a single end hole situated $2 \mathrm{~mm}$ from the catheter tip. A radio-opaque marker is sited $2 \mathrm{~mm}$ from the tip and suction is provided by hand with a lockable $20 \mathrm{ml}$ syringe. The catheter has previously been used as part of the PercuSurge thrombectomy system.

\section{CASE REPORT}

A 41 year old man was admitted for elective coronary catheterisation as part of the investigation of idiopathic dilated cardiomyopathy. Angiography was performed by the trainee undertaking basic cardiac catheterisation education. The right radial artery was cannulated with the Radifocus introducer II system (Terumo). According to routine procedure, the patient received an intra-arterial antispasm cocktail of $200 \mu \mathrm{g}$ of glyceryl trinitrate and $5 \mathrm{mg}$ of verapamil followed by $5000 \mathrm{U}$ of heparin. Standard JR4 and JL4 Impulse diagnostic catheters (Boston Scientific) were used. Catheterisation of the right coronary artery was uneventful showing a smooth, normal coronary artery. On engagement and initial injection of the left coronary artery, the patient began to experience severe chest pain and ST segments in V4-V6 began to rise, subsequently peaking at $3 \mathrm{~mm}$. Angiography showed a stump in the position of the second obtuse marginal (fig lA) with contrast slowly eddying in the proximal vessel (fig 1B). The patient was immediately given $100 \%$ oxygen and opiate analgesia. The diagnostic catheter was exchanged for a Kimny guiding catheter (Boston Scientific) and a further $5000 \mathrm{U}$ of heparin was given. A Galeo wire (Biotronik) was passed easily beyond the occlusion site and followed with the Export aspiration catheter. Aspiration was begun once the radio-opaque tip was in the obtuse marginal artery and continued to $3 \mathrm{~mm}$ proximal to the wire tip in three slow passes (fig lC).

A small quantity (less than $1 \mathrm{ml}$ ) of air was recovered in the syringe. The patient's symptoms rapidly disappeared and the ECG normalised. Angiography showed TIMI (thrombolysis in myocardial infarction) grade 3 flow into a branching distal territory with some spasm where the occlusion had been (fig 2A) that responded well to nitrates (fig 2B).
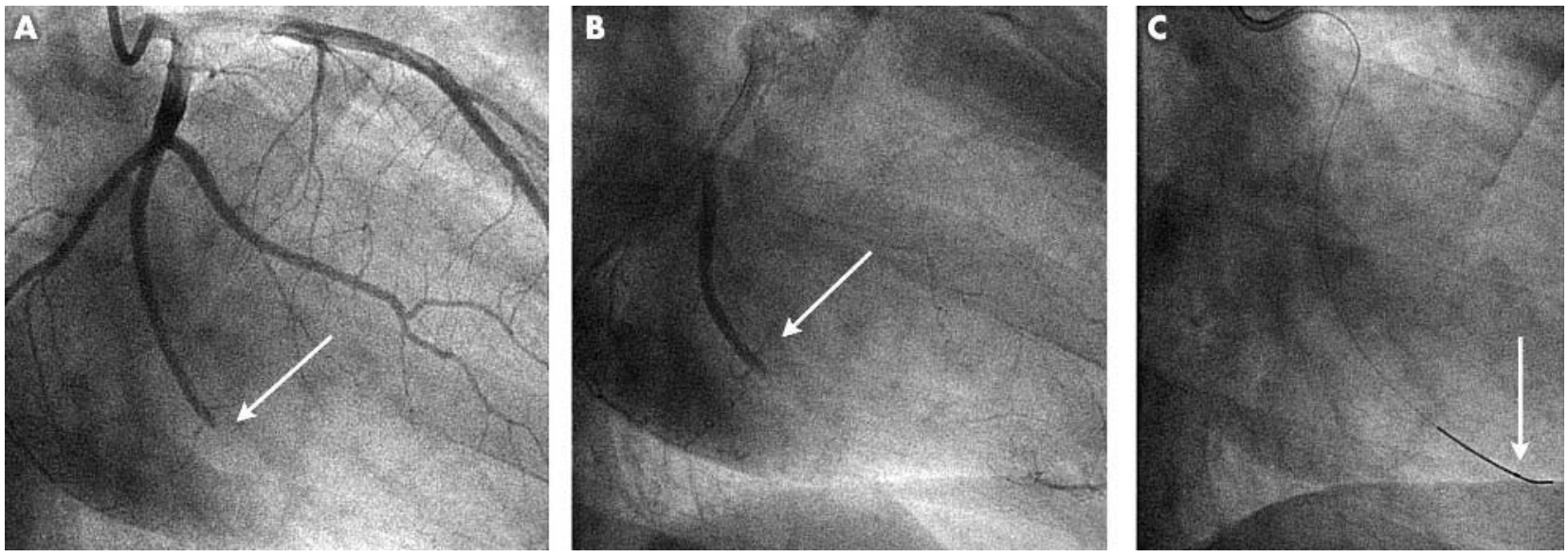

Figure 1 (A) Stump of obtuse marginal artery (arrow). (B) Dye stasis in occluded vessel (arrow). (C) Radio-opaque tip of aspiration catheter on guidewire distal to occlusion (arrow). 

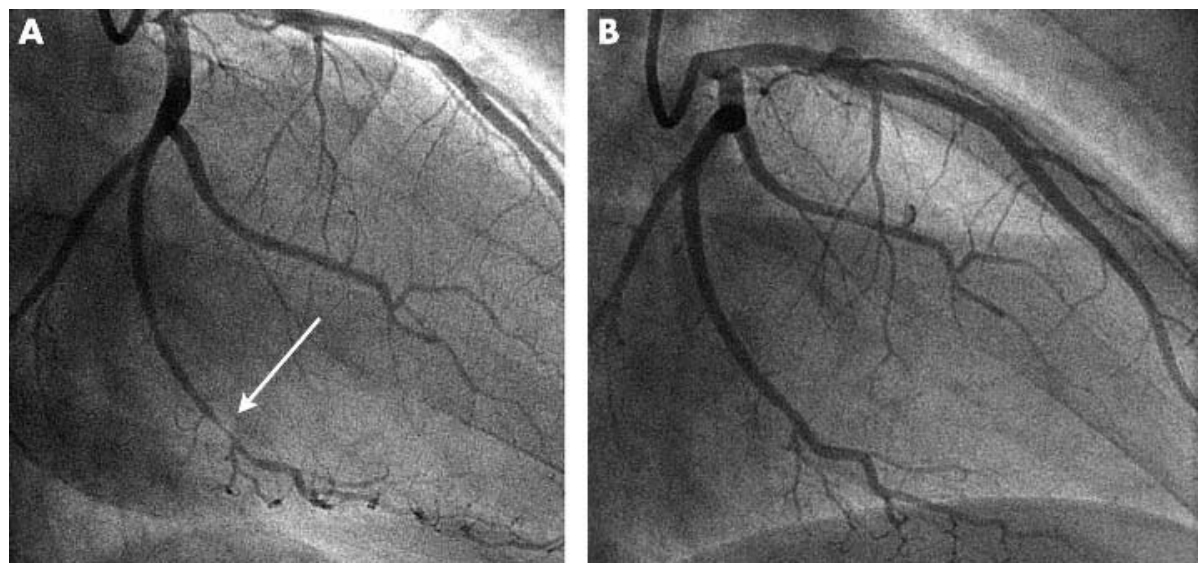

Figure 2 (A) Obtuse marginal artery reopened with spasm (arrow). (B) Spasm in obtuse marginal resolved after administration of nitrates.

The patient had an uneventful overnight stay and was discharged the following day with a normal ECG and cardiac enzymes.

\section{DISCUSSION}

Treatment for an air embolus has consisted of 100\% oxygen with the aim of accelerating air embolus reabsorption ${ }^{3}$ and analgesia.

Some case reports have described mechanical methods in the treatment of an air embolus. Disruption or dislodgement by the guidewire ${ }^{5}$ and the forceful injection of saline ${ }^{25}$ are aimed at fragmenting the air embolus to allow dispersal or to force it distally. Such interventions may result in main vessel patency but clearly such techniques have a potential to damage the main vessel during aggressive manoeuvres and to damage the distal microvasculature due to widespread, smaller embolisations. In contrast, aspiration aims at resolving the blockage by removing the air.

Aspiration has been attempted with non-dedicated devices such as over the wire (OTW) balloons ${ }^{36}$ or the angiography catheter itself. ${ }^{2}$ Clearly these techniques have several limitations. The lumen of such an OTW balloon system is just large enough to allow 0.014 inch wire passage and this may limit rapid aspiration. Once the wire is removed any forward movement of the system has an appreciable risk of damage or perforation, thus allowing only a single pullback with each deployment. Practical problems of deployment of OTW systems can occur compared with monorail systems and, lastly, such OTW systems have become less common in many catheterisation suites. Use of the angiography catheter as an aspiration tool is limited by the ability to manipulate it deeply enough to affect the embolus and the risk of damage while attempting this. In contrast the Export aspiration catheter is a dedicated system for coronary artery aspiration. As such it has the favourable qualities of being a monorail system that has the flexibility and slenderness to reach distal lesions down tortuous vessels and is amenable to repeated passes at the site where the embolus is lodged and, if necessary, distally. The aspiration catheter has a relatively large lumen of $1 \mathrm{~mm}$ (about three times the diameter of the lumen of an OTW balloon with concomitant capacity for increased flow) that allows relatively rapid flow for rapid removal of intracoronary debris or fluid.

Our case illustrates that the Export aspiration catheter is suitable for, and is worth consideration in, the treatment of coronary air embolus.

\section{Authors' affiliations}

M S Patterson, F Kiemeneii, Amsterdam Department of Interventional Cardiology, Onze Lieve Vrouwe Gasthuis, Amsterdam, the Netherlands

The authors have no financial or competing interests.

Correspondence to: Dr Mark S Patterson, Amsterdam Department of Interventional Cardiology, OLVG, le Oosterparkstraat 279, 1090 HM, Amsterdam, Netherlands; markspatterson@doctors.net.uk

Accepted 25 January 2005

\section{REFERENCES}

1 Dodek A, Boone JA, Hooper RO, et al. Complications of left coronary arteriography. Can Med Assoc J 1983;128:934-6.

2 Khan M, Schmidt DH, Bajwa T, et al. Coronary air embolism: incidence, severity, and suggested approaches to treatment. Cathet Cardiovasc Diagn 1995:36:313-8.

3 Solodky A, Birnbaum Y, Assali A, et al. Coronary air embolism treated by bubble aspiration. Catheter Cardiovasc Interv 2000;49:452-4.

4 Bentivoglio LG, Leo LR. Death from coronary air embolism during percutaneous transluminal coronary angioplasty. Cathet Cardiovasc Diagn 1985; 11:585-90.

5 Inove T, Yaguchi I, Mizoguchi K, et al. Air embolism in the right coronary artery occurring during the left coronary angioplasty using the guiding catheter with a side hole. Catheter Cardiovasc Interv 2000;49:331-4.

6 Haraphongse $M$, Rossall RE. Large air embolus complicating coronary amgioplasty. Cathet Cardiovasc Diagn 1989;17:168-71. 